\title{
Birds of Dhorpatan Hunting Reserve, Nepal
}

\author{
Saroj Panthi ${ }^{1 *}$, Sher Singh Thagunna ${ }^{2}$ \\ ${ }^{1}$ Department of National Park and Wildlife Conservation, Dhorpatan Hunting Reserve, Baglung, Nepal \\ ${ }^{2}$ Department of National Park and Wildlife Conservation, Api-Nampa Conservation Area, Darchula, Nepal \\ Email: "mountsaroj@gmail.com \\ Received June $18^{\text {th }}$, 2013; revised July 22 ${ }^{\text {nd }}, 2013$; accepted August $7^{\text {th }}, 2013$
}

\begin{abstract}
Copyright (c) 2013 Saroj Panthi, Sher Singh Thagunna. This is an open access article distributed under the Creative Commons Attribution License, which permits unrestricted use, distribution, and reproduction in any medium, provided the original work is properly cited.
\end{abstract}

\begin{abstract}
Nepal is rich in bird diversity and recorded 871 bird species. Our study aimed to update bird diversity of Dhorpatan Hunting Reserve (DHR) of Nepal which is an Important Birds Area (IBA) out of 27 IBAs of Nepal. One hundred forty nine species of birds were recorded in DHR; out of them Catreus wallichii was globally threatened bird which has faced different anthropogenic problem. Twelve species of birds were included in CITIES Appendices and 3 species were nationally threatened. Most of the species of order Passeriformis were recorded. Only one species of order Upupiformes i.e. Upupa epops was recorded during the study.
\end{abstract}

Keywords: Bird; Checklist; Threatened; Dhorpatan Hunting Reserve

\section{Introduction}

Birds (class Aves) are feathered, winged, bipedal, endothermic, egg-laying, vertebrate animals (Wikipedia, 2013). Although Nepal covers just $0.1 \%$ of the global land mass, nearly $9 \%$ of the world's bird species are found here (BCN, 2012). With the latest record of ashy minivet (Pericrocotus divaricatus) and long-billed vulture (Gypus indicus) Nepal's bird diversity has reached 871 species (BCN, 2012; BCN and DNPWC, 2012). A total of 29 species recorded in Nepal were identified as globally threatened by BirdLife International in 1999 (Grimmett et al., 2000). Zoo-geographically, Nepal falls between two great regions: the Palaeartic in the north and Oriental to the south so Nepal has one of the world's richest avian fauna (Shrestha, 1981).

A total of 27 Important Birds Areas (IBAs) had been identified in Nepal. Thirteen IBAs are within protected areas (Thakuri \& Thapa, 2012). Dhorpatan Hunting Reserve (DHR) is one of the IBAs (BCN and DNPWC, 2011). DHR was a habitat of 137 species of bird out of them cheer pheasant (Catreus wallichii) is listed in endangered category of IUCN red data list (Thapa, 2007). Here we updated the checklist of bird species of DHR. DHR was good habitat of birds (Thapa, 2007) but checklist update was lacking. Our aim was to update the checklist of bird species in DHR and to identify their order, family, conservation status and threats.

\section{Material and Methods}

\section{Study Area}

The Dhorpatan Hunting Reserve (DHR) is the only one hunting reserve in Nepal located in Dhaulagiri Himal range of the eastern part of the country (Aryal \& Kreigenhofer, 2009; Panthi et al., 2012). The reserve was established in 1983 and gazetted in 1987. The reserve covers $1325 \mathrm{~km}^{2}$ and ranges from 2000 to 7246 m (altitude). It falls within the Rukum, Baglung and

\footnotetext{
*Corresponding author.
}

Myadgi districts of Nepal (Figure 1). The reserve supports 14 ecosystems types represented in the mid hill to higher Himalayan ecosystem (Shrestha et al., 2002; Lillesø et al., 2005; Bhuju et al., 2007).

The reserve provide prime habitat for fauna such as barking deer (Munticus muntjak), Himalayan thar (Hemitragus jemlachicus), rhesus macaque (Macaca radiata) wolf (Canis lupus), red panda (Ailurus fulgens fulgens), wild boar (Sus scorfa), blue sheep (Pseudois nayaur), Himalayan black bear (Ursus thibetans), common leopard (Panthera pardus), goral (Naemorhedus goral), serow (Capricornis thar) and variety of avifauna including cheer pheasant (Catrus wallichii) and danphe (Lopophorus impegians) (Aryal \& Kreigenhofer, 2009; Aryal et al., 2010a; Aryal et al., 2010b; Panthi et al., 2012).

\section{Methods: Checklist of Birds in DHR}

Intensive survey was conducted throughout the reserve to update the checklist of birds in study area. We used The books "Birds of Nepal” prepared by Richard Grimmett, Carol Inskipp and Tim Inskipp (2000) and "Birds of Nepal: An Official Checklist” (2012) prepared by Bird Conservation of Nepal (BCN) and Department of National Parks and Wildlife Conservation (DNPWC) to identify the birds during the study. We visited all seven hunting blocks of DHR viz. Barse, Dogadi, Fagune, Ghustung, Seng, Sundaha and Surtibang blocks and observed the birds in two season viz. winter and summer throughout the year 2012. We observed the birds carefully by the help of binoculars took photographs with digital camera and compared that photo with the photos of book to identify the birds. We also interviewed local people and DHR staffs to indentify birds and their threats during the study.

\section{Results and Discussion}

We recorded one hundred forty nine species of birds in DHR (Table 1). Out of them, Catreus wallichii was globally threat- 


\section{S. PANTHI, S. S. THAGUNNA}

Table 1.

Bird of Dhorpatan Hunting Reserve (DHR), identified during the survey periods in 2012.

\begin{tabular}{|c|c|c|c|c|c|c|}
\hline \multirow{2}{*}{ S.N. } & \multirow{2}{*}{ Common name } & \multirow{2}{*}{ Scientific name } & \multirow{2}{*}{ Order } & \multirow{2}{*}{ Family } & \multirow{2}{*}{$\begin{array}{l}\text { Conservation } \\
\text { status }\end{array}$} & \multirow{2}{*}{$\begin{array}{c}\text { CITIES } \\
\text { Appendix }\end{array}$} \\
\hline & & & & & & \\
\hline 1 & Mallard & Anas platyrhynchos & Anseriformes & Anatidae & & \\
\hline 2 & Ruddy Shelduck & Tadorna ferruginea & Anseriformes & Anatidae & & \\
\hline 3 & Black Eagle & Ictinaetus malayensis & Ciconiformis & Accipitridae & & II \\
\hline 4 & Cinereous Vulture & Aegypius monachus & Ciconiformis & Accipitridae & & II \\
\hline 5 & Himalayan Griffon & Gyps himalayensis & Ciconiformis & Accipitridae & & II \\
\hline 6 & Pallas's Fish Eagle & Haliaeetus leucoryphus & Ciconiformis & Accipitridae & & II \\
\hline 7 & Ibisbill & Ididorhyncha struthersii & Ciconiformis & Charadriidae & & \\
\hline 8 & Common Krestrel & Falco tinnunculus & Ciconiformis & Falconidae & & II \\
\hline 9 & Little Stint & Calidris minuta & Ciconiformis & Scolopacidae & & \\
\hline 10 & Curlew Sandpiper & Calidris ferruginea & Ciconiformis & Scolopacidae & & \\
\hline 11 & Hill Pigeon & Columba rupestris & Columbiformes & Columbidae & & \\
\hline 12 & Oriental Turtle Dove & Streptopelia orientalis & Columbiformes & Columbidae & & \\
\hline 13 & Rock Pigeon & Columba livia & Columbiformes & Columbidae & & \\
\hline 14 & Snow Pigeon & Columba palumbus & Columbiformes & Columbidae & & \\
\hline 15 & Speckled Wood Pigeon & Columba hodgsonii & Columbiformes & Columbidae & & \\
\hline 16 & Spotted Dove & Streptopelia chinensis & Columbiformes & Columbidae & & \\
\hline 17 & Blood Pheasant & Ithaginis cruentus & Galliformis & Phasianidae & & II \\
\hline 18 & Cheer Pheasant & Catreus wallichii & Galliformis & Phasianidae & GT, NP & I \\
\hline 19 & Hill Partridge & Arborophila torqueola & Galliformis & Phasianidae & & \\
\hline 20 & Himalayan Monal & Lophophorus impejanus & Galliformis & Phasianidae & NP & I \\
\hline 21 & Kalij Pheasant & Lophura leucomelanos & Galliformis & Phasianidae & & \\
\hline 22 & Koklas Pheasant & Pucrasia macrolopha & Galliformis & Phasianidae & & \\
\hline 23 & Satyr Tragopan & Tragopan satyra & Galliformis & Phasianidae & NP & III \\
\hline 24 & Snow Patridge & Lerwa lerwa & Galliformis & Phasianidae & & \\
\hline 25 & White-Throated Tit & Aegithalos niveogularis & Passeriformes & Aegithalidae & & \\
\hline 26 & Oriental Skylark & Alauda gulgula & Passeriformes & Alaudidae & & \\
\hline 27 & Winter Wren & Troglodytes troglodytes & Passeriformes & Certhiidae & & \\
\hline 28 & Brown Dipper & Cinclus pallasii & Passeriformes & Cinclidae & & \\
\hline 29 & Hill Prinia & Prinia atrogularis & Passeriformes & Cisticolidae & & \\
\hline 30 & Striated Prinia & Prinia criniger & Passeriformes & Cisticolidae & & \\
\hline 31 & Zitting Cisticola & Cisticola juncidis & Passeriformes & Cisticolidae & & \\
\hline 32 & Ashy Drongo & Dicrurus leucophaeus & Passeriformes & Corvidae & & \\
\hline 33 & Common Raven & Corvus corax & Passeriformes & Corvidae & & \\
\hline 34 & Common Iora & Aegithina tiphia & Passeriformes & Corvidae & & \\
\hline
\end{tabular}




\begin{tabular}{|c|c|c|c|c|}
\hline 35 & Eurasian Golden Oriole & Oriolus oriolus & Passeriformes & Corvidae \\
\hline 36 & House Crow & Corvus splendes & Passeriformes & Corvidae \\
\hline 37 & Large-Billed Crow & Corvus macrorhynchos & Passeriformes & Corvidae \\
\hline 38 & Rufous Treepie & Dendrocitta vagabunda & Passeriformes & Corvidae \\
\hline 39 & Short-Billed Minivet & Pericrocotus flammeus & Passeriformes & Corvidae \\
\hline 40 & Spotted Nutcracker & Nucifraga caryocatactes & Passeriformes & Corvidae \\
\hline 41 & Yellow-Billed Blue Magpie & Urocissa flavirostris & Passeriformes & Corvidae \\
\hline 42 & Yellow-Billed Chough & Pyrrhocorax graculus & Passeriformes & Corvidae \\
\hline 43 & Yellow-Billed Fantail & Rhipidura hypoxantha & Passeriformes & Corvidae \\
\hline 44 & White-Throated Fantail & Rhipidura albicollis & Passeriformes & Corvidae \\
\hline 45 & Beautiful Rosefinch & Carpodacus pulcherrimus & Passeriformes & Fringillidae \\
\hline 46 & Blanford's Rosefinch & Carpodacus rubescens & Passeriformes & Fringillidae \\
\hline 47 & Collard Grosbeak & Mycerobas affinis & Passeriformes & Fringillidae \\
\hline 48 & Common Rosefinch & Carpodacus erythrinus & Passeriformes & Fringillidae \\
\hline 49 & Creasted Bunting & Melophus lathami & Passeriformes & Fringillidae \\
\hline 50 & Dark-Rumpled Rosefinch & Carpodacus edwardsii & Passeriformes & Fringillidae \\
\hline 51 & Fire-Fronted Serin & Serinus pusillus & Passeriformes & Fringillidae \\
\hline 52 & Little Bunting & Emberiza pusilla & Passeriformes & Fringillidae \\
\hline 53 & Pine Bunting & Emberiza leucocephalos & Passeriformes & Fringillidae \\
\hline 54 & Pink-Browed Rosefinch & Carpodacus rodochrous & Passeriformes & Fringillidae \\
\hline 55 & Red-Fronted Rosefinch & Carpodacus puniceus & Passeriformes & Fringillidae \\
\hline 56 & Rock Bunting & Emberiza cia & Passeriformes & Fringillidae \\
\hline 57 & Scarlet Finch & Haematospiza sipahi & Passeriformes & Fringillidae \\
\hline 58 & Streaked Rosefinch & Carpodacus rubicilloides & Passeriformes & Fringillidae \\
\hline 59 & Twite & Carduelis flavirostris & Passeriformes & Fringillidae \\
\hline 60 & Vinaceous Rosefinch & Carpodacus vinaceus & Passeriformes & Fringillidae \\
\hline 61 & White-Winged Grosbeak & Mycerobas carnipes & Passeriformes & Fringillidae \\
\hline 62 & Yellow-Breasted Greenfinch & Carduelis spinoides & Passeriformes & Fringillidae \\
\hline 63 & Barn Swallow & Hirundo rustica & Passeriformes & Hirundinidae \\
\hline 64 & Black-Backed Forktail & Enicurus immaculatus & Passeriformes & Muscicapidae \\
\hline 65 & Black Redstrat & Phoenicurus ochruros & Passeriformes & Muscicapidae \\
\hline 66 & Blue-Capped Redstrat & Phoenicurus coeruleocephalus & Passeriformes & Muscicapidae \\
\hline 67 & Blue-Fronted Redstrat & Phoenicurus frontalis & Passeriformes & Muscicapidae \\
\hline 68 & Common Stonechat & Saxicola torquata & Passeriformes & Muscicapidae \\
\hline 69 & Dark-Sided Flycatcher & Muscicapa sibirica & Passeriformes & Muscicapidae \\
\hline 70 & Dark-Thorated Thrush & Turdus ruficollis & Passeriformes & Muscicapidae \\
\hline 71 & Dusky Thrush & Turdus naumanni & Passeriformes & Muscicapidae \\
\hline
\end{tabular}


S. PANTHI, S. S. THAGUNNA

Continued

\begin{tabular}{|c|c|c|c|c|}
\hline 72 & Eurasian Blackbird & Turdus merula & Passeriformes & Muscicapidae \\
\hline 73 & Golden Bush Robin & Tarsiger chrysenus & Passeriformes & Muscicapidae \\
\hline 74 & Grandala & Grandala coelicolor & Passeriformes & Muscicapidae \\
\hline 75 & Grey Bushchat & Saxicola ferra & Passeriformes & Muscicapidae \\
\hline 76 & Grey-Winged Blackbird & Turdus boulboul & Passeriformes & Muscicapidae \\
\hline 77 & Hodgson's Redstrat & Phoenicurus hodgosni & Passeriformes & Muscicapidae \\
\hline 78 & Indian Blue Robin & Luscinia brunnea & Passeriformes & Muscicapidae \\
\hline 79 & Kessler's Thrush & Turdus kessleri & Passeriformes & Muscicapidae \\
\hline 80 & Mistle Thrush & Turdus viscivorus & Passeriformes & Muscicapidae \\
\hline 81 & Orange-Flanked Bush Robin & Tarsiger cyanurus & Passeriformes & Muscicapidae \\
\hline 82 & Orental Magpie Robin & Copsychus saularis & Passeriformes & Muscicapidae \\
\hline 83 & Pied Bushchat & Saxicola caprata & Passeriformes & Muscicapidae \\
\hline 84 & Plumbeous Water Redstart & Rhyacornis fuliginosus & Passeriformes & Muscicapidae \\
\hline 85 & Purple Cochoa & Cochoa purpurea & Passeriformes & Muscicapidae \\
\hline 86 & Red-Thorated Flycatcher & Ficedula parva & Passeriformes & Muscicapidae \\
\hline 87 & Rufous-Brested Bush Robin & Tarsiger hyperythrus & Passeriformes & Muscicapidae \\
\hline 88 & Rufous-Gorgeted Flycatcher & Ficedula strophiata & Passeriformes & Muscicapidae \\
\hline 89 & Rusty-Tailed Flycatcher & Muscicapa ruficauda & Passeriformes & Muscicapidae \\
\hline 90 & White-Browed Bush Robin & Tarsiger indicus & Passeriformes & Muscicapidae \\
\hline 91 & White-Bellied Redstrat & Hodgsonius phaenicuroides & Passeriformes & Muscicapidae \\
\hline 92 & White-Capped Water Redstart & Chaimarronis leucocephalus & Passeriformes & Muscicapidae \\
\hline 93 & White-Collard Blackbird & Turdus albocinctus & Passeriformes & Muscicapidae \\
\hline 94 & White-Gorgeted Flycatcher & Ficedula monileger & Passeriformes & Muscicapidae \\
\hline 95 & White-Tailed Robin & Myiomela leucura & Passeriformes & Muscicapidae \\
\hline 96 & White-Throated Redstrat & Phoenicurus schisticeps & Passeriformes & Muscicapidae \\
\hline 97 & Black-Lored Tit & Parus xanthogenys & Passeriformes & Paridae \\
\hline 98 & Coal Tit & Parus ater & Passeriformes & Paridae \\
\hline 99 & Fire-Capped Tit & Cephalopyrus flammiceps & Passeriformes & Paridae \\
\hline 100 & Green-Backed Tit & Parus monticolus & Passeriformes & Paridae \\
\hline 101 & Rufous-Napped Tit & Parus rufonuchalis & Passeriformes & Paridae \\
\hline 102 & Rufous-Vented Tit & Parus rubidiventris & Passeriformes & Paridae \\
\hline 103 & Alpine Accentor & Prunella collaris & Passeriformes & Passeridae \\
\hline 104 & Altai Accentor & Prunella himalayana & Passeriformes & Passeridae \\
\hline 105 & Brown Accentor & Prunella fulvescens & Passeriformes & Passeridae \\
\hline 106 & Citrine Wagtail & Motacilla citreola & Passeriformes & Passeridae \\
\hline 107 & Grey Wagtail & Motacilla cinerea & Passeriformes & Passeridae \\
\hline 108 & House Sparrow & Passer domesticus & Passeriformes & Passeridae \\
\hline
\end{tabular}




\begin{tabular}{|c|c|c|c|c|}
\hline 109 & Olive-Backed Pipit & Anthus hodgsoni & Passeriformes & Passeridae \\
\hline 110 & Paddyfield Pipit & Anthus rufulus & Passeriformes & Passeridae \\
\hline 111 & Red-Thorated Pipit & Anthus cervinus & Passeriformes & Passeridae \\
\hline 112 & Robin Accentor & Prunella rubeculoides & Passeriformes & Passeridae \\
\hline 113 & Rosy Pipit & Anthus rosestus & Passeriformes & Passeridae \\
\hline 114 & Tree Pipit & Anthus trivialis & Passeriformes & Passeridae \\
\hline 115 & Upland Pipit & Anthus sylvanus & Passeriformes & Passeridae \\
\hline 116 & Yellow Wagtail & Motacilla flava & Passeriformes & Passeridae \\
\hline 117 & Long-Tailed Shrike & Lanius schach & Passeriformes & Pittidae \\
\hline 118 & Black Bulbul & Hypsipetes leucocephalus & Passeriformes & Pycnonotidae \\
\hline 119 & Himalayan Bulbul & Pycnonotus leucogenys & Passeriformes & Pycnonotidae \\
\hline 120 & Striated Bulbul & Pycnonotus melanicterus & Passeriformes & Pycnonotidae \\
\hline 121 & Goldcrest & Regulus regulus & Passeriformes & Regulidae \\
\hline 122 & Black-Faced Laughinthrush & Garrulax affinis & Passeriformes & Sylviidae \\
\hline 123 & Black-Throated Parrotbill & Paradoxornis niplensis & Passeriformes & Sylviidae \\
\hline 124 & Brownish-Flanked Bush Warbler & Cettia fortipes & Passeriformes & Sylviidae \\
\hline 125 & Chesnut-Crowned Bush Warbler & Cettia major & Passeriformes & Sylviidae \\
\hline 126 & Great Parrotbill & Conostoma oemodium & Passeriformes & Sylviidae \\
\hline 127 & Greenish Warbler & Phylloscopus trochiloides & Passeriformes & Sylviidae \\
\hline 128 & Grey-Hooded Warbler & Seicercus xanthoschistos & Passeriformes & Sylviidae \\
\hline 129 & Hoary-Thorated Barwing & Actinodura nipalensis & Passeriformes & Sylviidae \\
\hline 130 & Hume’s Warbler & Phylloscopus humei & Passeriformes & Sylviidae \\
\hline 131 & Jungle Babbler & Turdoides striatus & Passeriformes & Sylviidae \\
\hline 132 & Large-Billed Leaf Warbler & Phylloscopus magnirostris & Passeriformes & Sylviidae \\
\hline 133 & Rufus-Vented Yuhina & Yuhina occipitalis & Passeriformes & Sylviidae \\
\hline 134 & Slender-Billed Scimitar Babbler & Xiphirhynchus Superciliaris & Passeriformes & Sylviidae \\
\hline 135 & Straited Laughinthrush & Garrulax striatus & Passeriformes & Sylviidae \\
\hline 136 & Streaked Laughinthrush & Garrulax lineatus & Passeriformes & Sylviidae \\
\hline 137 & Variegated Laughinthrush & Garrualx variegates & Passeriformes & Sylviidae \\
\hline 138 & Whistler's Warbler & Seicercus whistleri & Passeriformes & Sylviidae \\
\hline 139 & White-Browed Tit Warbler & Leptopoecile sophiae & Passeriformes & Sylviidae \\
\hline 140 & White-Creasted Laughingthrush & Garrulax monileger & Passeriformes & Sylviidae \\
\hline 141 & White-Throated Laughingthrush & Garrulax albogularis & Passeriformes & Sylviidae \\
\hline 142 & Yellow-Bellied Warbler & Abroscopus supercilliaris & Passeriformes & Sylviidae \\
\hline 143 & Crimson-Brested Woodpecker & Dendrocopos cathpharius & Piciformes & Indicatoridae \\
\hline 144 & Greater Flameback & Chrysocolaptes lucidus & Piciformes & Indicatoridae \\
\hline 145 & Rufous-Bellied Woodpecker & Dendrocopos hyperythrus & Piciformes & Indicatoridae \\
\hline
\end{tabular}




\begin{tabular}{lcclll}
\hline 146 & Little Owl & Athene noctua & Strigiformes & Strigidae & II \\
147 & Spotted Owlet & Athene brama & Strigiformes & Strigidae & II \\
148 & Tawny Owl & Strix aluco & Strigiformes & Strigidae & II \\
149 & Common Hoopoe & Upupa epops & Upupiformes & Upupidae & \\
\hline
\end{tabular}

Note: GT: Globally Threatened, NP: Nationally Protected.

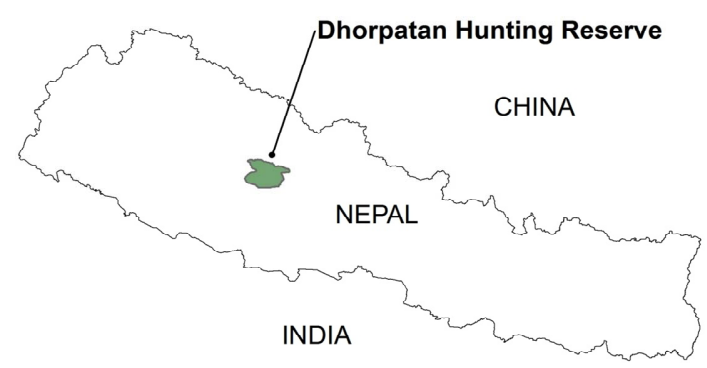

Figure 1.

Dhorpatan hunting reserve, Nepal.

ened bird which has faced different anthropogenic problem.

C. wallichii Lophophorus impejanus Tragopan satyra are nationally protected birds found in the reserve. C. wallichii and $L$. impejanus are included in CITIES appendix I (BCN and DNPWC 2012).

We recorded 9 species which are included in CITIES appendix II and one species which is included in CITIES appendix III. Most of the species of order Passeriformis were recorded. Only one species of order Upupiformes i.e. Upupa epops was recorded during the study.

This study showed that snares were main threats of birds. Local people used snares to capture large sized birds such as Arborophila torqueola, Catreus wallichii, Ithaginis cruentus Lophophorus impejanus, Lophura leucomelanos, Pucrasia macrolopha and Tragopan satyra for fulfillment of meat.

We recorded snares in two places viz. Karichaur in Fagune block and Rukhacahur in Barse block. Small children killed small sized forest birds due to lack of awareness. The present study provided basic information on birds of DHR. However further species wise information is needed. Distribution and detail threats identification of globally threatened and nationally protected species are further recommended study in DHR.

\section{Acknowledgements}

The study was possible after the financial and logistic support from the DHR office and Department of National Parks and Wildlife Conservation (DNPWC). We thanked to all staffs of DHR and DNPWC who helped for field survey. We also thank to Dr. Achyut Aryal (Institute of Natural and Mathematical Sciences, Massey University, New Zealand) for his guidance to complete this study.

\section{REFERENCES}

Aryal, A., \& Kreigenhofer, B. (2009). Summer diet composition of the common leopard Panthera pardus (Carnivora: Felidae) in Nepal. Journal of Threatened Taxa, 1, 562-566. http://dx.doi.org/10.11609/JoTT.02287.562-6

Aryal, A., Gastaur, S., Menzel, S., Chhetri, T. B., \& Hopkins, J. (2010a). Estimation of blue sheep population parameters in the Dhorpatan Hunting Reserve, Nepal. International Journal of Biodiversity and Conservation, 2, 51-56.

Aryal, A., Sathyakumar, S., \& Kreigenhofer, B. (2010b). Opportunistic animal's diet depends on prey availability: Spring dietary composition of the red fox (Vulpes vulpes) in the Dhorpatan Hunting Reserve, Nepal. Journal of Ecology and the Natural Environment, 2, 59-63.

Bhuju, U. R., Shakya, P. R., Basnet, T. B., \& Shrestha, S. (2007). Nepal biodiversity resource book: Protected areas, ramsar sites, and world heritage sites. ICIMOD and MOEST, Government of Nepal in cooperation with UNEP, Kathmandu.

Bird Conservation Nepal (2012). Annual Report 2011-12: Bird conservation Nepal, Lazimpat Kathmandu Nepal.

Bird Conservation of Nepal and Department of National Parks and Wildlife Conservation (2012). Birds of Nepal: An Official Checklist, Kathmandu, Nepal.

Bird Conservation of Nepal and Department of National Parks and Wildlife conservation (2011). The state of Nepal's birds 2010: Bird conservation of Nepal and Department of National Parks and Wildlife Conservation, Kathmandu Nepal.

Grimmett, R., Inskipp, C., \& Inskipp, T. (2000). Birds of Nepal: Christopher Helm, an imprint of A\& C Black Publishers Ltd., 36 Soho Square, London WID 3QY.

Lillesø, J. P. B., Shrestha, T. B., Dhakal, L. P., Nayaju, R. P., \& Shrestha, R. (2005). The map of potential vegetation of Nepal-A forestry/agroecological/biodiversity classification system: Forest \& Landscape. Development and Environment Series 2-2005 and CFC-TIS Document Series No.110. Denmark.

Panthi, S., Aryal, A., Lord, J., Adhikari B., \& Raubenheimer, D. (2012). Summer diet and habitat ecology of red panda (Ailurus fulgens fulgens) in Dhopatan Hunting Reserve, Nepal. Zoological Studies, 51, 701-709.

Shrestha, T. B., Lillesø, J. P. B., Dhakal, L. P., \& Shrestha, R. (2002). Forest and vegetation types of Nepal: MFSC, HMG/Nepal, Natural Resource Management Sector Assistance Programme (NARMSAP) and Tree Improvement and Silviculture Component (TISC), Kathmandu.

Shrestha, T. K., (1981). Wildlife of Nepal. Kathmandu: Curriculum Development Centre Tribhuvan University.

Thakuri, J. J., \& Thapa, I. (2012). Assessment of Reshunga forest for an important bird area status. Danphe, 21, 1-4.

Thapa, B. B. (2007). Dhorpatan sikar aararksha ko parichaya. Baglung, Nepal: Department of National Park and Wildlife Conservation, DHR Office.

Wegge, P. (1976). Himalayan shikar reserves; surveys and management proposals. Field Document No. 5. FAO/NEP/72/002 Project, Kathmandu, $96 \mathrm{p}$.

Wikipedia (2013). Birds. http://en.wikipedia.org/wiki/List_of_birds_of_Nepal 